\title{
The path integral for the statistical sum of the microcanonical ensemble in cosmology
}

\author{
A.O.Barvinsky ${ }^{1,2}$ \\ ${ }^{1}$ Theory Department, Lebedev Physics Institute, Leninsky Prospect 53, Moscow 119991, Russia \\ ${ }^{2}$ Department of Physics, Ludwig Maximilians University, Theresienstrasse 37, Munich, Germany
}

\begin{abstract}
The path integral is calculated for the statistical sum of the microcanonical ensemble in a generic time-parametrization invariant gravitational model with the Friedman-Robertson-Walker (FRW) metric. This represents the first example of a systematic calculation of the Faddeev-Popov gauge-fixed path integral in the minisuperspace sector of quantum cosmology. The gauge fixing procedure, together with gauging out local diffeomorphisms, also handles the residual symmetries associated with the conformal Killing vector of the FRW metric and incorporates the BatalinVilkovisky quantization technique for gauge theories with linearly dependent generators. For a subset of saddle-point instantons, characterized by a single oscillation of the FRW scale factor, this technique is designed to obtain the one-loop statistical sum in the recently suggested model of cosmological initial conditions generated by a conformal field theory with a large number of quantum species.
\end{abstract}

\section{Introduction}

The purpose of this paper is to calculate beyond tree-level approximation the path integral for the statistical sum in the generic time-parametrization invariant gravitational system with the FriedmanRobertson-Walker (FRW) metric. This problem is motivated by the recently suggested model of initial conditions in cosmology in the form of the microcanonical density matrix [1, 2. In contrast to the formal Euclidean quantum gravity origin of the no-boundary prescription 3 . or essentially semiclassical tunneling prescription 4 for the cosmological state, this model has a clear origin in terms of operator quantization of gravity theory in the Lorentzian signature spacetime. In particular, it is based on a natural notion of the microcanonical density matrix as a projector on the space of solutions of the quantum gravitational Dirac constraints - the system of Wheeler-DeWitt equations [2]. Moreover, when applied to cosmology with a large number of fields conformally coupled to gravity, this theory can be important within the cosmological constant and dark energy problems. In particular, its statistical ensemble is bounded to a finite range of values of the effective cosmological constant, it generates an inflationary stage and is potentially capable of generating the cosmological acceleration phenomenon within the so-called Big Boost scenario [5].

As shown in 2], for a spatially closed cosmology with $S^{3}$-topology the microcanonical statistical sum can be represented by the Euclidean quantum gravity path integral,

$$
Z=\int_{\text {periodic }} D\left[g_{\mu \nu}, \phi\right] e^{-S\left[g_{\mu \nu}, \phi\right]},
$$

over the metric $g_{\mu \nu}$ and matter fields $\phi$ which are periodic on the Euclidean spacetime with a compactified time $\tau$ (of $S^{1} \times S^{3}$ topology). The FRW metric arises in this path integral as the set of major collective variables of cosmology. Under the decomposition of the full set of $g_{\mu \nu}(x), \phi(x)$ into the minisuperspace FRW sector

$$
d s^{2}=N^{2}(\tau) d \tau^{2}+a^{2}(\tau) d^{2} \Omega^{(3)},
$$


and inhomogeneous "matter" fields $\Phi(x)=\left(\phi(x), \psi(x), A_{\mu}(x), h_{\mu \nu}(x), \ldots\right)$ on the background of this metric the path integral can be cast into the form of an integral over a minisuperspace lapse function $N(\tau)$ and a scale factor $a(\tau)$,

$$
\begin{aligned}
& Z=\int D[a, N] e^{-\Gamma[a, N]}, \\
& e^{-\Gamma[a, N]}=\int D \Phi(x) e^{-S[a, N ; \Phi(x)]} .
\end{aligned}
$$

Here, $\Gamma[a, N]$ is the Euclidean effective action of the fields $\Phi$ (which include also the metric perturbations $h_{\mu \nu}$ ) on the FRW background, and $S[a, N ; \Phi(x)] \equiv S_{E}\left[g_{\mu \nu}, \phi\right]$ is the original action rewritten in terms of this minisuperspace decomposition. It is important that this representation is not a minisuperspace approximation, when all the fields $\Phi(x)$ are frozen out. Rather this is the disentangling of the collective degrees of freedom from the configuration space, the rest of which effectively manifests itself in terms of this effective action.

In the theory with a primordial cosmological constant and a large number of free (linear) fields conformally coupled to gravity - conformal field theory (CFT) - such effective action is dominated by the contribution of these fields because they simply outnumber the non-conformal fields including, in particular, the graviton. Then this quantum effective action is exactly calculable as a functional of histories $(a(\tau), N(\tau))$ by the conformal transformation converting (1.2) into the static (Einstein Universe) metric with $a=$ const $[6,7,8]$. The structure of the resulting action [1] is

$$
\begin{aligned}
& \Gamma[a, N]=\oint d \tau N \mathcal{L}\left(a, a^{\prime}\right)+F(\eta), \\
& \eta=\oint d \tau \frac{N}{a} .
\end{aligned}
$$

where $a^{\prime} \equiv d a / N d \tau$ and the integration runs over the period of $\tau$ on the circle $S^{1}$ of $S^{1} \times S^{3}$. Here the effective Lagrangian of its local part $\mathcal{L}\left(a, a^{\prime}\right)$ includes the classical Einstein term and the polarization effects of quantum fields and their vacuum (Casimir) energy. A nonlocal part of the action $F(\eta)$ is the free energy of their quasi-equilibrium excitations with the temperature given by the inverse of the conformal time (1.6). This is a typical boson or fermion sum $F(\eta)= \pm \sum_{\omega} \ln \left(1 \mp e^{-\omega \eta}\right)$ over field oscillators with energies $\omega$ on a unit 3 -sphere. In what follows the concrete structure of $\mathcal{L}\left(a, a^{\prime}\right)$ and $F(\eta)$ will be unimportant for us - remarkably the one-loop functional integration can be done in a closed form without specifying a concrete form of these functions. The only restriction will be the absence of higher derivatives of $a$ in $\mathcal{L}\left(a, a^{\prime}\right)$, which of course implies a special UV renormalization of the effective action (1.4). Such a renormalization really exists, as was shown for the CFT driven cosmology 1, and it does not introduce into the minisuperspace sector of Einstein theory extra degrees of freedom 1$]$

Semiclassically the integral (1.3) is dominated by the saddle points - solutions of the effective Friedmann equation of the Euclidean gravity theory, which for a generic Lagrangian of the above type reads as

$$
\frac{\delta \Gamma}{\delta N}=\mathcal{L}-\frac{\partial \mathcal{L}}{\partial a^{\prime}} a^{\prime}+\frac{1}{a} \frac{d F}{d \eta}=0
$$

The term with $d F / d \eta$ here characterizes the energy of the gas of thermally excited particles with the inverse temperature $\eta$ - the instanton period in units of the conformal time. The latter is given by the integral (1.6) over the full period of $\tau$ or the $2 k$-multiple of the integral between the two neighboring turning points of the scale factor history $a(\tau), a^{\prime}\left(\tau_{ \pm}\right)=0$.

\footnotetext{
${ }^{1}$ This choice of renormalization was motivated in [1] by ghost-free requirements and certain universality properties which, in particular, relate the value of the Casimir energy to the coefficient the conformal anomaly of CFT fields - the source of $\mathcal{L}\left(a, a^{\prime}\right)$ in (1.5) 9, 10.
} 
This $k$-fold nature follows from the fact that in a periodic solution of (1.7) the scale factor necessarily oscillates certain number of times between its maximum and minimum values $a_{ \pm}=a\left(\tau_{ \pm}\right)$, $a_{-} \leq a(\tau) \leq a_{+}$, and forms a kind of a garland of $S^{1} \times S^{3}$ topology with oscillating $S^{3}$ sections. These garland-type instantons are weighted in the relevant statistical ensemble by their exponentiated onshell action

$$
Z=P \exp \left(-\Gamma_{0}\right),
$$

where $\Gamma_{0}=\Gamma[a, N]$ is taken at the solution of Eqs.(1.6)-(1.7) and $P$ is the preexponential factor accumulating quantum corrections of the semiclassical expansion. For a particular case of the CFT driven cosmology [1, these instantons exist only in the limited range of the cosmological constant $\Lambda=3 H^{2}, 0<H_{\min }^{2}<H^{2}<H_{\max }^{2}$ and, thus, might be relevant to the solution of the cosmological constant problem [1, 2. Here we will focus on the calculation of the one-loop prefactor $P$ for a generic action (1.5). Application of this calculation to the CFT driven cosmology will be considered in a sequel to this paper [11.

In fact, this represents the first example of a systematic and explicit one-loop calculation of the gauge-fixed path integral in the minisuperspace sector of quantum cosmology. Compared to early examples of the path integral calculation, that were basically focused on the formal derivation of the Wheeler-DeWitt equations [12, 13, 14, 15, or applications to cosmological toy models [17, our results here apply to the models with the effective minisuperspace action of a rather generic form (1.5) which includes according to (1.4) the effect of inhomogeneous field modes. Therefore, this is not a minisuperspace approximation freezing out all local inhomogeneous degrees of freedom. Moreover, our calculations of the statistical sum differ by boundary conditions - periodic boundary conditions vs the Dirichlet (or mixed Neumann-Dirichlet 18) boundary conditions for the unitary transition amplitude of 13, 14, 15, 16. This leads to an additional peculiarity of the formalism which finally amounts to a special gauge fixing procedure - the one for the system with linearly dependent generators of gauge transformations - and, thus, goes beyond conventional Faddeev-Popov technique.

The nature of this peculiarity is as follows. By counting the number of local degrees of freedom the minisuperspace sector of $a$ and $N$ is not dynamical (a second order action with two-dimensional configuration space and one local gauge invariance), and the one-loop prefactor $P$ seems being a trivial normalization constant. This conclusion is, however, misleading because of the global degree of freedom related to the periodic nature of $S^{1} \times S^{3}$ and its interplay with the zero mode associated with the conformal Killing symmetry of a generic FRW metric (1.2). Gauging this symmetry out effectively leads to the model with linearly dependent generators, which requires the use of the BatalinVilkovisky quantization technique [19]. Application of this technique shows that the cancelation in the preexponential factor, associated with the absence of local degrees of freedom, has only a partial nature and leaves us with a nontrivial contribution to $P$ of the CFT radiation bath (namely its specific heat $d^{2} F / d \eta^{2}$ ).

Below we calculate this contribution for a subset of background instantons, corresponding to the solutions of (1.7) with a single oscillation of the scale factor $a(\tau)$. After a brief formulation of main results in Sect.2, we begin this one-loop calculation in Sect.3 with the derivation of the quadratic part of the action, which turns out to be parameterized by a single function $g(\tau)$ - the zero mode of the operator of small disturbances on the instanton background. Then in Sect.4 we describe the gauge fixing procedure which reveals the residual conformal Killing invariance of the action and incorporates the set of linearly dependent generators. The necessary Batalin-Vilkovisky technique [19] is then briefly presented in Sect.5. In Sects.6 and 7 we calculate the contributions of the gauge and ghost sectors of the path integral and the sector of metric perturbations, which lead to the final closed algorithm for $P$. We accomplish the paper with concluding remarks in Conclusions. Two appendices contain the derivation of gauge independence properties in the Batalin-Vilkovisky technique for systems with linearly dependent generators and the treatment of a redundant set of gauge conditions by means of the so-called extraghost [19]. 


\section{The formulation of main results}

The action (1.5)-(1.6) with the Lagrangian $\mathcal{L}\left(a, a^{\prime}\right)$ - a rather generic function of $a$ and its parametrization invariant derivative $a^{\prime} \equiv d a / N d \tau$ - is invariant with respect to local reparametrizations of time, $\tau \rightarrow \bar{\tau}=\bar{\tau}(\tau)$,

$$
\bar{a}(\bar{\tau})=a(\tau), \quad \bar{N}(\bar{\tau})=\left(\frac{d \bar{\tau}}{d \tau}\right)^{-1} N(\tau) .
$$

As we show below, the gauge-fixed path integral (1.3) for the statistical sum of this model can be written down in the one-loop approximation in terms of a special set of variables $\varphi$ and $n$ as

$$
\begin{aligned}
Z=e^{-\Gamma_{0}} \int D n \delta\left[n^{\prime}(\tau)\right] & \left(\operatorname{Det}_{*} \boldsymbol{Q}\right) \\
& \times \int D \varphi \delta(\oint d \tau g \varphi) Q \exp \left(-\Gamma_{(2)}[\varphi, n]\right) .
\end{aligned}
$$

Here the delta function of the gauge condition $n^{\prime}$ and the relevant Faddeev-Popov functional determinant $\operatorname{Det}_{*} \boldsymbol{Q}$ gauge out the local time-parametrization invariance of the action $\Gamma_{(2)}[\varphi, n]$ quadratic in its arguments. The star denotes the removal of the residual gauge invariance with respect to the conformal Killing transformations: the symbol of the restricted functional determinant $\operatorname{Det}_{*} \boldsymbol{Q}$ implies the omission of the relevant zero mode of $\boldsymbol{Q}$. This residual gauge transformation is the invariance of $\Gamma_{(2)}[\varphi, n]$ under the global transformation $\varphi(\tau) \rightarrow \varphi(\tau)+\Delta^{\varepsilon} \varphi(\tau), \Delta^{\varepsilon} \varphi(\tau) \equiv \varepsilon g(\tau)$, with the function $g(\tau)$ - the zero mode of the operator of field disturbances $\varphi$,

$$
\begin{aligned}
& \boldsymbol{F}=\frac{\delta^{2} \Gamma_{(2)}}{\delta \varphi(\tau) \delta \varphi\left(\tau^{\prime}\right)}, \\
& \boldsymbol{F} g=0 .
\end{aligned}
$$

It is gauged out in the integral over $\varphi$ by the delta function of the (time-nonlocal) gauge condition $\chi[\varphi]=\oint d \tau g \varphi$, which implies a functional orthogonality of $\varphi(\tau)$ to the zero mode $g(\tau)$. This is accompanied by the Faddeev-Popov factor $Q, \Delta^{\varepsilon} \chi \equiv Q \varepsilon$,

$$
Q=\oint d \tau g^{2}(\tau) \text {. }
$$

The Gaussian integration over $\varphi$ and the integration over $n(\tau)$, which in view of the gauge condition $n^{\prime}=0$ reduces to the integral over the constant mode $n_{0}=$ const, give the preexponential factor of $(2.2)$

$$
P=\text { const } \times\left(\operatorname{Det}_{*} \boldsymbol{F}\right)^{-1 / 2} \int d n_{0} \exp \left(n_{0}^{2} \frac{Y}{\boldsymbol{I}}\right),
$$

where the coefficient $Y$ differs from unity by the contribution of the specific heat $d^{2} F(\eta) / d \eta^{2}$ of conformal modes, whereas the restricted functional determinant $\operatorname{Det}_{*} \boldsymbol{F}$ (with its zero mode gauged out) is given exactly by the factor $\boldsymbol{I}$ which is contained above in the denominator of the exponential - the property proven in the accompanying paper [20,

$$
\begin{aligned}
& Y=1-\boldsymbol{I} \frac{d^{2} F}{d \eta^{2}}, \\
& \operatorname{Det}_{*} \boldsymbol{F}=\text { const } \times \boldsymbol{I} .
\end{aligned}
$$

Therefore, after Gaussian integration over $n_{0}$ the resulting one-loop prefactor $P$ differs from a trivial normalization constant only by the contribution of matter sector $O\left(d^{2} F / d \eta^{2}\right)$ to $Y$ and reads

$$
P=\frac{\text { const }}{\sqrt{|Y|}}
$$


The details of the above mechanism are as follows. To begin with, the new variables, which parameterize the perturbations of the scale factor $\delta a$ and the lapse function $\delta N$ on the background of the solution of the equation of motion for (1.5), read as

$$
\begin{aligned}
& \delta a=\frac{a a^{\prime}}{g} \varphi, \\
& \delta N=\frac{a^{\prime}}{g} \varphi+n a .
\end{aligned}
$$

Here the function $g=g(\tau)$ - the zero mode introduced above - expresses in terms of the Hessian of the Lagrangian with respect to the scale factor "velocity" $a^{\prime}$,

$$
g=a^{\prime} a \sqrt{|\mathcal{D}|}, \quad \mathcal{D}=\frac{\partial^{2} \mathcal{L}}{\partial a^{\prime} \partial a^{\prime}} .
$$

The most important property of these variables is that $\varphi$ is canonically normalized, and that the local part of their quadratic action has a simple closed form which is universally parametrized by the same single function $g(\tau)$,

$$
\begin{aligned}
& \Gamma_{(2)}[\varphi, n]=\frac{1}{2} \varepsilon_{\mathcal{D}} \oint d \tau\left\{\varphi^{\prime 2}+\frac{g^{\prime \prime}}{g} \varphi^{2}+\left(4 n g^{\prime}+2 n^{\prime} g\right) \varphi+g^{2} n^{2}\right\} \\
& \quad+\frac{1}{2} \frac{d^{2} F}{d \eta^{2}}(\oint d \tau n)^{2} \\
& \varepsilon_{\mathcal{D}}=\frac{\mathcal{D}}{|\mathcal{D}|}= \pm 1 .
\end{aligned}
$$

This property holds irrespective of the form of the Lagrangian $\mathcal{L}\left(a, a^{\prime}\right)$, and the source of this universality is, of course, the time-parametrization invariance of the action and the fact that $\mathcal{L}\left(a, a^{\prime}\right)$ does not contain higher order derivatives of $a$.

The operator (2.3) for this action equals

$$
\boldsymbol{F}=-\frac{d^{2}}{d \tau^{2}}+\frac{g^{\prime \prime}}{g} .
$$

It has as the zero mode the periodic regular function $g(\tau)$. Other important properties of this operator follow from the following observations.

The one-fold instanton solution (which only we consider in this paper) has one oscillation of the scale factor between its maximal and minimal values $a_{ \pm}=a\left(\tau_{ \pm}\right)$. Therefore, the function $g(\tau) \sim a^{\prime}(\tau)$ has two zeroes at these points, $g\left(\tau_{ \pm}\right)=0$, which mark the boundaries of the half period of the total time range, $T=2\left(\tau_{+}-\tau_{-}\right)$. For brevity of the formalism we shift the point of the minimal $a_{-}$to zero, $\tau_{-}=0$, and let the coordinate $\tau$ run in the total range $-\tau_{+} \leq \tau \leq \tau_{+}$with the points $\pm \tau_{+}$identified. Then $g(\tau)$ is an odd function of $\tau$ which is periodic with all its derivatives and has two first degree zeros at antipodal points $\tau=\tau_{-} \equiv 0$ and $\tau=\tau_{+}$of this circle

$$
\begin{aligned}
& g(\tau)=-g(-\tau), \\
& g\left(\tau_{ \pm}\right)=0, \quad g^{\prime}\left(\tau_{ \pm}\right) \equiv g_{ \pm}^{\prime} \neq 0 .
\end{aligned}
$$

In spite of singularity of $1 / g$ at $\tau_{ \pm}$the operator (2.15) is everywhere regular (analytic) on the circle, because from the equation of motion (1.7) it follows that all odd order derivatives of $a$ at $\tau_{ \pm}$vanish, and $g^{\prime \prime}\left(\tau_{ \pm}\right)=0$ (this is guaranteed by the assumption that $\mathcal{L}\left(a, a^{\prime}\right)$ is an even function of $a^{\prime}$ ).

The final result of this paper - the prefactor (2.7)-(2.9) - is determined by the second solution of the homogeneous equation $\boldsymbol{F} \Psi=0$, which together with $g(\tau)$ forms a full set of basis functions of $\boldsymbol{F}$. This is a two-point function $\Psi\left(\tau, \tau_{*}\right)$

$$
\Psi\left(\tau, \tau_{*}\right) \equiv g(\tau) \int_{\tau_{*}}^{\tau} \frac{d y}{g^{2}(y)}, \quad \tau_{-} \equiv 0<\tau<\tau_{+}, \quad \tau_{-}<\tau_{*}<\tau_{+},
$$


with some fixed point $\tau_{*}$ in the half period range of the instanton time. This function is smoothly defined only in the half-period range of $\tau$ and $\tau_{*}$, because otherwise the integral for $\Psi\left(\tau, \tau_{*}\right)$ is divergent if the roots of $g(\tau)$ lie between $\tau$ and $\tau_{*}$. Therefore (2.18) cannot be smoothly continued beyond the half-period $\tau_{-} \leq \tau \leq \tau_{+}$, though its limits are well defined for $\tau \rightarrow \tau_{ \pm} \mp 0$,

$$
\Psi\left(\tau_{ \pm}, \tau_{*}\right)=-\frac{1}{g^{\prime}\left(\tau_{ \pm}\right)} \equiv-\frac{1}{g_{ \pm}^{\prime}}
$$

because the factor $g(\tau)$ tending to zero compensates for the divergence of the integral at $\tau \rightarrow \tau_{ \pm}$.

The quantity $\boldsymbol{I}$ which determines the one-loop prefactor of our statistical sum (2.9)-(2.7) reads in terms of $\Psi$ as

$$
\begin{aligned}
& \boldsymbol{I}=2 \varepsilon_{\mathcal{D}}\left(\Psi_{+} \Psi_{+}^{\prime}-\Psi_{-} \Psi_{-}^{\prime}\right) \\
& \Psi_{ \pm} \equiv \Psi\left(\tau_{ \pm}, \tau_{*}\right), \quad \Psi_{ \pm}^{\prime} \equiv \Psi^{\prime}\left(\tau_{ \pm}, \tau_{*}\right) .
\end{aligned}
$$

Because of $g^{\prime \prime}\left(\tau_{ \pm}\right)=0$ the function $\Psi\left(\tau, \tau_{*}\right)$ is differentiable at $\tau \rightarrow \tau_{ \pm}$, and all the quantities which enter this expression are well defined. These properties of $\Psi\left(\tau, \tau_{*}\right)$ guarantee that (2.20) is independent of an arbitrary choice of the point $\tau_{*}$, which can be easily verified by using a simple relation $d \Psi_{ \pm}^{\prime} / d \tau_{*}=$ $-g_{ \pm}^{\prime} / g^{2}\left(\tau_{*}\right)$.

\section{Quadratic part of the action}

The quadratic part of the action can be simplified by a systematic use of equations of motion for the background (1.7) and

$$
\frac{1}{N} \frac{\delta \Gamma}{\delta a}=\frac{\partial \mathcal{L}}{\partial a}-\left(\frac{\partial \mathcal{L}}{\partial a^{\prime}}\right)^{\prime}-\frac{1}{a^{2}} \frac{d F}{d \eta}=0,
$$

and their time derivatives (note that (3.1) is the derivative of (1.7) which is the manifestation of the time-parametrization invariance). Thus, after integration by parts, the second order variation in $\delta a$ of the local part of the action (1.5) can be transformed by using the differentiated (and devided by $\left.a^{\prime}\right)$ version of (3.1),

$$
\delta_{a}^{2} \Gamma=\oint d \tau\left\{\mathcal{D}\left(\delta a^{\prime}\right)^{2}+\frac{1}{a^{\prime}}\left(\mathcal{D} a^{\prime \prime}\right)^{\prime} \delta a^{2}\right\}+\frac{d^{2} F}{d \eta^{2}}\left(\delta_{a} \eta\right)^{2},
$$

where $\mathcal{D}$ is defined by (2.12),$\delta_{a} \eta=-\oint d \tau \delta a / a^{2}$ and the background value of the lapse function was chosen to be $N=1$.

The functional $\Gamma=\Gamma[a, N]$ is invariant with respect to the linearized version of the one-dimensional diffeomorphism (2.1), $\bar{\tau}=\tau+f(\tau)$, with the periodic parameter $f(\tau)$ on a circle $-\tau_{-} \leq \tau \leq \tau_{+}$,

$$
\begin{aligned}
& \Delta^{f} \delta N \equiv \overline{\delta N}(\tau)-\delta N(\tau)=-f^{\prime}, \\
& \Delta^{f} \delta a \equiv \overline{\delta a}(\tau)-\delta a(\tau)=-a^{\prime} f .
\end{aligned}
$$

Therefore it satisfies the following Ward identity

$$
\frac{\delta \Gamma}{\delta a}=\frac{N}{a^{\prime}}\left(\frac{\delta \Gamma}{\delta N}\right)^{\prime}
$$

which allows one to simplify on shell (1.7) the mixed $\delta N \delta a$-variation of the action,

$$
\delta_{N} \delta_{a} \Gamma=\oint d \tau N\left(\delta_{N} \frac{\delta \Gamma}{\delta N}\right)^{\prime} \frac{\delta a}{a^{\prime}}=-\oint d \tau\left(\frac{\delta a}{a^{\prime}}\right)^{\prime}\left(\mathcal{D} a^{\prime 2}\right) \delta N+\frac{d^{2} F}{d \eta^{2}} \delta_{N} \eta \delta_{a} \eta
$$


where $\delta_{N} \eta=\oint d \tau \delta N / a$. Here the integration by parts in the first term is admissible because the derivative of the singular at $a^{\prime}=0$ quantity $\delta a / a^{\prime}$ is compensated by the factor $a^{\prime 2}$.

Similarly

$$
\delta_{N}^{2} \Gamma=\delta_{N} \oint d \tau\left(\mathcal{L}-\frac{\partial \mathcal{L}}{\partial a^{\prime}} a^{\prime}+\frac{1}{a} \frac{d F}{d \eta}\right) \delta N=\oint d \tau\left(\mathcal{D} a^{\prime 2}\right) \delta N^{2}+\frac{d^{2} F}{d \eta^{2}}\left(\delta_{N} \eta\right)^{2},
$$

and the second order variation of the action in terms of perturbations $\delta a$ and $\delta N$ finally takes the form

$$
\begin{aligned}
& \Gamma_{(2)}=\frac{1}{2} \delta^{2} \Gamma=\frac{1}{2} \oint d \tau\left\{\mathcal{D}\left(\delta a^{\prime}\right)^{2}+\frac{1}{a^{\prime}}\left(\mathcal{D} a^{\prime \prime}\right)^{\prime} \delta a^{2}\right. \\
&\left.-2\left(\frac{\delta a}{a^{\prime}}\right)^{\prime}\left(\mathcal{D} a^{\prime 2}\right) \delta N+\left(\mathcal{D} a^{\prime 2}\right) \delta N^{2}\right\}+\frac{1}{2} \frac{d^{2} F}{d \eta^{2}}(\delta \eta)^{2}, \\
& \delta \eta=\delta_{N} \eta+\delta_{a} \eta= \oint d \tau\left(\frac{\delta N}{a}-\frac{\delta a}{a^{2}}\right) .
\end{aligned}
$$

Formal integration by parts allows one to convert the integral of the first three terms to the quadratic form

$$
\frac{1}{2} \oint d \tau\left(\mathcal{D} a^{\prime 2}\right) \Psi^{2}, \quad \Psi \equiv \delta N-\left(\frac{\delta a}{a^{\prime}}\right)^{\prime}
$$

in the variable $\Psi$ which is a local invariant of the linearized diffeomorphism transformation.2, (3.3)(3.4). This representation is however illegitimate because the integrand of this form is not integrable in the vicinity of points with $a^{\prime}=0$, these divergences being acquired via divergent total derivative terms. The action simplifies in terms of another set of variables which were introduced in Sect.2 by Eqs.(2.10)-(2.11) with the function $g=g(\tau)$ given by (2.12).

In terms of these variables the variation of this global degree of freedom - the conformal time period of the instanton - reads as

$$
\delta \eta=\oint d \tau n
$$

and, as one can easily check, the local part of the quadratic action (3.8) after a number of nonsingular integrations by parts takes the form

$$
\begin{gathered}
\frac{1}{2} \oint d \tau\left\{\mathcal{D}\left(\delta a^{\prime}\right)^{2}+\frac{1}{a^{\prime}}\left(\mathcal{D} a^{\prime \prime}\right)^{\prime} \delta a^{2}-2\left(\frac{\delta a}{a^{\prime}}\right)^{\prime}\left(\mathcal{D} a^{\prime 2}\right) \delta N+\left(\mathcal{D} a^{2}\right) \delta N^{2}\right\} \\
=\frac{1}{2} \varepsilon_{\mathcal{D}} \oint d \tau\left\{\varphi^{\prime 2}+\frac{g^{\prime \prime}}{g} \varphi^{2}+\left(4 n g^{\prime}+2 n^{\prime} g\right) \varphi+g^{2} n^{2}\right\} .
\end{gathered}
$$

Thus we come to the final elegant form of the quadratic action (2.13), which turns out to be functionally parameterized by a single function $g$.

\section{The choice of gauge conditions}

\subsection{Admissibility of relativistic gauges}

In terms of the new variables $\phi=(\varphi, n)$ the diffeomorphism transformations (3.3)-(3.4) take the form

$$
\Delta^{f} n=-\tilde{f}^{\prime}, \quad \Delta^{f} \varphi=-g \tilde{f}, \quad \tilde{f} \equiv \frac{f}{a}
$$

\footnotetext{
${ }^{2}$ This is a long wavelength version of the gauge-invariant variable of cosmological perturbations [21] 22$]$
} 
with the rescaled parameter of gauge transformations $\tilde{f}$. The gauge condition $\chi=\chi(\phi)$ should be such that any field $\phi$ can be transformed to the representative of the gauge orbit (denoted by the bar, $\bar{\phi}$ ) which satisfies this gauge. In other words the equation $0=\bar{\chi} \equiv \chi+\Delta^{f} \chi$ should always have a unique solution $f$ for any $\phi$, which means that the Faddeev-Popov operator $Q=Q(d / d \tau)$ in $\Delta^{f} \chi \equiv Q f$ should be invertible.

Though locally the equation for $f$ might be solvable, there can be global obstructions to the existence of such a solution. For example, take the gauge on the Lagrangian multiplier not containing its time derivative, $\chi=n$. The transition to this gauge is achieved via the gauge parameter solving the equation $\tilde{f}^{\prime}=n$, which is however not periodic because of an obvious jump $\tilde{f}(T)-\tilde{f}(0)=\oint d \tau n$. On the contrary, the relativistic gauge containing a time derivative of $n$, like $\chi=n^{\prime}$, has a larger freedom in boundary conditions admitting periodic solutions for diffeomorphisms. Indeed, the solution of $\tilde{f}^{\prime \prime}=n^{\prime}$,

$$
\tilde{f}(\tau)=\int_{0}^{\tau} d \tau_{1} n_{1}+C \tau+\tilde{f}(0)
$$

is periodic under the following choice of the integration constant $C=-\oint d \tau_{1} n_{1} / T$.

\subsection{Special gauge: positive definiteness of the Euclidean action}

The gauge fixing procedure generally can affect the form of the action and, in particular, change the convexity property of its quadratic part in the sector of gauge degrees of freedom. The action (2.13) prior to gauge fixing is not positive/negative definite, because the potential term $g^{\prime \prime} / g$ in the quadratic form in $\varphi$ is basically negativ 3 , and the operator $\boldsymbol{F}$ is indefinite. This can be improved by using the gauge of the form

$$
\chi=\frac{g^{\prime \prime}}{g} \varphi+2 n g^{\prime}+n^{\prime} g=0 .
$$

Such a gauge is admissible because $\Delta^{f} \chi=(g f / a)^{\prime \prime}$, and its Faddeev-Popov operator

$$
Q(d / d \tau) f=\frac{d^{2}}{d \tau^{2}} \frac{g f}{a}
$$

is invertible under the condition of regularity. Indeed, the only periodic zero mode of this operator $f \propto a / g$ is singular at $\tau_{ \pm}$and should be discarded. Therefore, $Q$ has a regular periodic Green's function on a circle. With this gauge imposed the quadratic action (2.13) takes the form with the reversed sign of $\left(g^{\prime \prime} / g\right) \varphi^{2}$, whereas all the other terms become definite in sign (positive or negative depending on $\varepsilon_{\mathcal{D}}= \pm 1$ )

$$
\Gamma_{(2)}=\frac{1}{2} \varepsilon_{\mathcal{D}} \oint d \tau\left\{\varphi^{\prime 2}-\frac{g^{\prime \prime}}{g} \varphi^{2}+g^{2} n^{2}\right\}+\frac{1}{2} \frac{d^{2} F}{d \eta^{2}}(\oint d \tau n)^{2} .
$$

In our concrete model of the CFT driven cosmology we have

$$
\varepsilon_{\mathcal{D}}=-1, \quad \frac{d^{2} F}{d \eta^{2}}<0, \quad \frac{g^{\prime \prime}}{g}<0
$$

and the action becomes negative definite for real $n$ and $\varphi$. With $\varphi$ expressed via the gauge (4.3) as

$$
\varphi=-\frac{\left(n g^{2}\right)^{\prime}}{g^{\prime \prime}}
$$

\footnotetext{
${ }^{3}$ Remember that $g(\tau) \sim a^{\prime}(\tau)$ is an oscillating function, and its convexity in the average is opposite to the sign of its amplitude
} 
this provides a well defined procedure of Gaussian integration over $n$ along the imaginary axis contour $n \in[-i \infty,+i \infty]$. This is dictated by the original definition of the microcanonical path integral in the physical spacetime with the Lorentzian signature [2, 23. Indeed, the Euclidean path integral (1.3) is the transformed version of the path integral over Lorentzian signature metrics for the microcanonical statistical sum in cosmology [2, 23. This definition implies the integration over imaginary values of the Euclidean gravity lapse function and signifies an imaginary $n$ - the alternative $((3+1)$-noncovariant $)$ version of the so-called conformal rotation in Euclidean quantum gravity designed to render its Einstein action positive-definite [24].

Unfortunately, the action (4.5) with (4.7) has fourth-order derivatives and is hard to handle. Therefore we consider below another relativistic gauge. It does not improve the convexity properties of the action (and thus makes the choice of the path integration contour trickier), but renders the problem tractable from the calculational point of view.

\subsection{The relativistic gauge and residual gauge transformations}

In what follows we will use the relativistic gauge

$$
\chi \equiv n^{\prime}=0
$$

in which the variable $n$ becomes constant, $n=n_{0}=$ const, and the action simplifies to

$$
\begin{aligned}
\Gamma_{(2)}=\frac{1}{2} \varepsilon_{\mathcal{D}} \oint d \tau\{ & \left.\varphi^{\prime 2}+\frac{g^{\prime \prime}}{g} \varphi^{2}+4 n_{0} g^{\prime} \varphi\right\} \\
& +\frac{1}{2} \varepsilon_{\mathcal{D}} n_{0}^{2} \oint d \tau g^{2}+\frac{1}{2} \frac{d^{2} F}{d \eta^{2}} n_{0}^{2} T^{2},
\end{aligned}
$$

where $T$ denotes the full period of the instanton

$$
T=\oint d \tau \text {. }
$$

This gauge does not fix the gauge freedom completely, because it remains invariant under the residual gauge transformations (4.1) with a special parameter $f(\tau)=-a(\tau) \varepsilon, \varepsilon=$ const. It has a simple geometric interpretation - conformal Killing symmetry of a generic FRW background. Indeed, with this choice of $f$ the diffeomorphism of $\delta a$ and $\delta N$ (3.3)-(3.4) coincides with the local conformal transformation of these perturbations on the background of $(a(\tau), N=1)$

$$
\Delta^{\omega} \delta a(\tau)=\omega(\tau) a(\tau), \quad \Delta^{\omega} \delta N(\tau)=\omega(\tau) N=\omega(\tau)
$$

with the conformal factor parameter $\omega(\tau)=a^{\prime}(\tau) \varepsilon$ This explains the origin of extra symmetry not fixed by the coordinate gauge $n^{\prime}=0$ - conformally non-invariant Einstein and anomaly parts of the action (1.5) become invariant under those conformal transformations which coincide with diffeomorphisms, and this occurs for the conformal Killing transformation which exists for any FRW metric.

The parameter $f(\tau)=-a(\tau) \varepsilon$ is regular and periodic, and therefore this residual gauge freedom cannot be ruled out by boundary conditions, but rather has to be fixed by an extra gauge condition. As we will see now this procedure leads to the problem of quantization of the theory with linearly dependent generators.

In order to formulate the full set of resulting gauges we first collect all variables and the parameters of transformations - original local diffeomorphisms $\Delta^{f} \phi^{i}$ and residual global diffeomorphisms $\Delta^{\varepsilon} \phi^{i} \equiv$

\footnotetext{
${ }^{4}$ With another choice of time in the FRW metric, $N \neq 1$, these transformations are modified by factors of $N$, and the conformal Killing diffeomorphism is given by $f=-\varepsilon a / N$.
} 
$\left.\Delta^{f} \phi^{i}\right|_{f=-a \varepsilon}-$ into their multiplets

$$
\phi^{i}=\left[\begin{array}{l}
\varphi(\tau) \\
n(\tau)
\end{array}\right], \quad f^{\alpha}=\left[\begin{array}{c}
f(\tau) \\
\varepsilon
\end{array}\right] .
$$

Here the condensed indices $i$ and $\alpha$ include the continuous entry $\tau$ along with the discrete entry of the above 2 -dimensional columns. In these notations the full set of transformations reads as $\Delta^{f} \phi^{i}=R_{\alpha}^{i} f^{\alpha}$, where the generators $R_{\alpha}^{i}$ form the functional matrix

$$
R_{\alpha_{2}}^{i_{1}}=\left[\begin{array}{cc}
-\frac{g_{1} \delta\left(\tau_{1}, \tau_{2}\right)}{a_{2}} & g_{1} \\
-\frac{d}{d \tau_{1}} \frac{\delta\left(\tau_{1}, \tau_{2}\right)}{a_{2}} & 0
\end{array}\right],
$$

in which numerical labels of condensed indices imply different time coordinates $\tau_{1}$ and $\tau_{2}$ of their continuous entries. The contraction over $\alpha$ in $\Delta^{f} \phi^{i}=R_{\alpha}^{i} f^{\alpha}$ implies the integration over continuous time entries of $\alpha$ along with the summation over its discrete entries. The generators of both transformations $\Delta^{f} \phi^{i_{1}}=\oint d \tau_{2} R_{f_{2}}^{i_{1}} f_{2}$ and $\Delta^{\varepsilon} \phi^{i_{1}}=R_{\varepsilon}^{i_{1}} \varepsilon$ (with $R_{f_{2}}^{i_{1}}$ and $R_{\varepsilon}^{i_{1}}$ given by the first and the second column of the matrix (4.13) ) obviously form a linearly dependent set satisfying

$$
\oint d \tau_{2} R_{f_{2}}^{i_{1}} a\left(\tau_{2}\right)+R_{\varepsilon}^{i_{1}}=0 .
$$

In condensed notations this relation reads as

$$
R_{\alpha}^{i} Z^{\alpha}=0 .
$$

with the coefficients

$$
Z^{\alpha}=\left[\begin{array}{c}
a(\tau) \\
1
\end{array}\right] .
$$

As an additional gauge condition fixing the residual gauge transformation one can take a (functional) orthogonality of the quantum field $\varphi$ to the $\varepsilon$-transformation of $\Delta^{\varepsilon} \varphi$. This is the following global gauge condition

$$
\oint d \tau g \varphi=0
$$

which of course is admissible because the relevant Faddeev-Popov operator $Q$, characterizing the transformation of this gauge condition $\Delta^{\varepsilon} \oint d \tau g \varphi=Q \varepsilon$, is given by (2.5) and is invertible for any identically nonvanishing function $g$.

This global gauge together with the local gauge conditions (4.8) forms the following set

$$
\chi^{\alpha}=\left[\begin{array}{c}
n^{\prime}(\tau) \\
\oint d \tau g \varphi
\end{array}\right]
$$

which gives rise to the full block-structure Faddev-Popov operator, $\Delta^{f} \chi^{\alpha}=Q_{\beta}^{\alpha} f^{\beta}$,

$$
Q_{\beta}^{\alpha}=\left[\begin{array}{cc}
-\frac{d^{2}}{d \tau_{1}^{2}} \frac{\delta\left(\tau_{1}, \tau_{2}\right)}{a_{2}} & 0 \\
-\frac{g_{2}^{2}}{a_{2}} & Q
\end{array}\right] .
$$


This operator, $Q_{\beta}^{\alpha}=\left(\delta \chi^{\alpha} / \delta \phi^{i}\right) R_{\beta}^{i}$, is degenerate, because of the linear dependence of generators (4.15) and has as right zero-eigenvalue eigenvector the coefficients $Z^{\alpha}$

$$
Q_{\beta}^{\alpha} Z^{\beta}=0 .
$$

Inevitable left zero-eigenvalue eigenvector of this operator follow from the properties of the full set of gauge conditions (4.18). In view of $\oint d \tau n^{\prime}=0$ this set, similarly to the generators, is redundant and satisfies the functional linear dependence relation $\bar{Z}_{\alpha} \chi^{\alpha}=0$ with

$$
\bar{Z}_{\alpha}=\left[\begin{array}{ll}
1 & 0
\end{array}\right] .
$$

Here, as above, contraction of condensed indices in the time-dependent entry of the full index $\alpha=(\tau, \varepsilon)$ includes the $\tau$-integration. These coefficients form the left zero-eigenvalue eigenvector of (4.19)

$$
\bar{Z}_{\alpha} Q_{\beta}^{\alpha}=0 .
$$

A regular technique of handling gauge theories with linear dependent generators and redundant sets of gauge conditions is known as a part of the BV formalism [19, and we briefly present it in the next section.

\section{BV formalism for reducible gauge theories: one-loop ap- proximation}

The BV formalism of [19 suggests a quantization method for a generic gauge theory whose action $S[\phi]$ is invariant under gauge transformations with linearly dependent generators $R_{\alpha}^{i}$

$$
\begin{aligned}
& \frac{\delta S[\phi]}{\delta \phi^{i}} R_{\alpha}^{i}=0, \\
& R_{\alpha}^{i} Z_{a}^{\alpha}=0 .
\end{aligned}
$$

The coefficients of these linear dependence relations $Z_{a}^{\alpha}$ are labeled by a condensed index $a$. For simplicity we present here the case when all gauge fields, parameters of gauge transformations and these zero vectors $Z_{a}^{\alpha}$ are bosonic classically commuting variables and when these zero vectors are linearly independent themselves (the first-stage reducibility with the rank of the matrix $Z_{a}^{\alpha}$ coinciding with the range of the index $a$ ). The general case of boson-fermion systems of arbitrary stage of reducibility is fully considered in [19].

The gauge invariance of the action implies imposing the set of gauges

$$
\chi^{\alpha}=\chi^{\alpha}(\phi), \quad \bar{Z}_{\alpha}^{a} \chi^{\alpha}=0
$$

which should also be redundant not to overconstrain the system, which is actually invariant under the set of transformations whoes number is less than the range of index $\alpha$ - the rank of the matrix $R_{\alpha}^{i}$. Thus the numbers of zero vectors of generators and zero vectors of constraints $\bar{Z}_{\alpha}^{a}$ (the range of the index $a$ ) should coincide. The usual Faddeev-Popov operator for this set of gauges

$$
Q_{\beta}^{\alpha}=\frac{\delta \chi^{\alpha}}{\delta \phi^{i}} R_{\beta}^{i}
$$

is itself degenerate and has as right and left zero vectors $Z_{b}^{\beta}$ and $\bar{Z}_{\alpha}^{a}$ respectively. This means that the Faddeev-Popov ghosts $\left(C^{\beta}, \bar{C}_{\alpha}\right)$ which should generate $\operatorname{Det} Q_{\beta}^{\alpha}$ in the gauge-fixed path integral also become gauge fields and require auxiliary gauge fixing. Moreover, the redundancy of gauge conditions implies that their formal delta function $\delta[\chi]=\prod_{\alpha} \delta\left[\chi^{\alpha}\right] \sim \delta[0]$ is not well-defined and requires regularization. 
For the first-stage reducible theories this quantum gauge-fixing procedure is achieved by a special extension of the set of ghost fields and Lagrange multipliers [19]

$$
\Phi_{\mathrm{g}}=\left(C^{\alpha} ; \bar{C}_{\alpha}, \pi_{\alpha}\right) \rightarrow \Phi_{\mathrm{g}}=\left(C^{\alpha}, C^{a} ; \bar{C}_{\alpha}, \pi_{\alpha}, \bar{C}_{a}, \pi_{a}, E^{a}, P^{a}\right)
$$

in the effective (gauge-fixed) action $S_{\text {eff }}\left[\phi^{i}, \Phi_{\mathrm{g}}\right]$ and the corresponding path integral

$$
\begin{aligned}
& Z=\int D \phi D \Phi_{\mathrm{g}} \exp \left(-S_{\mathrm{eff}}\right) \\
& \begin{aligned}
S_{\mathrm{eff}}\left[\phi^{i}, \Phi_{\mathrm{g}}\right] & =S+\bar{C}_{\alpha} Q_{\beta}^{\alpha} C^{\beta}+\bar{C}_{a}\left(\omega_{\alpha}^{a} Z_{b}^{\alpha}\right) C^{b} \\
& +\left(\chi^{\alpha}+\sigma_{a}^{\alpha} E^{a}\right) \pi_{\alpha}+\pi_{a} \omega_{\alpha}^{a} C^{\alpha}+\bar{C}_{\alpha} \sigma_{a}^{\alpha} P^{a} .
\end{aligned}
\end{aligned}
$$

Here $\left(\bar{C}_{\alpha}, C^{\beta}, \pi_{a}, P^{a}\right)$ are Grassman anti-commuting variables whereas the rest are bosonic ones. The meaning of additional Lagrange multipliers $\pi_{a}$ and $P^{a}$ is that they generate delta functions of gauge conditions $\omega_{\alpha}^{a} C^{\alpha}$ and $\bar{C}_{\alpha} \sigma_{a}^{\alpha}$ for the original Faddeev-Popov ghosts $\left(C^{\alpha}, \bar{C}_{\alpha}\right)$ which now are gauge fields themselves. $\omega_{\alpha}^{a}$ and $\sigma_{a}^{\alpha}$ are arbitrary parameters of these gauges. The variables $C^{b}$ and $\bar{C}_{a}$ are the corresponding ghosts for the ghost $C^{\alpha}$. Finally, the integration over the Lagrange multiplier $\pi_{\alpha}$ of the original Faddeev-Popov scheme generates the delta function of the gauges $\chi^{\alpha}+\sigma_{a}^{\alpha} E^{a}$ which are modified by the contribution of the so-called extraghost $E^{a}$ [19. These modified gauges are no longer linearly dependent, which makes their delta function well-defined.

Thus, the integration over the ghost sector gives

$$
\begin{aligned}
\int D \Phi_{\mathrm{g}} \exp (- & \left.S_{\mathrm{eff}}\right) \\
& =\exp (-S) \operatorname{Det}\left[\begin{array}{cc}
Q_{\beta}^{\alpha} & \sigma_{b}^{\alpha} \\
\omega_{\beta}^{a} & 0
\end{array}\right] \frac{1}{\operatorname{Det}\left(\omega_{\alpha}^{a} Z_{b}^{\alpha}\right)} \int D E^{a} \delta\left[\sigma_{a}^{\alpha} E^{a}+\chi^{\alpha}\right] \\
& =\exp (-S) \frac{\operatorname{Det} \mathcal{F}_{\beta}^{\alpha}}{\operatorname{Det} q_{b}^{a} \operatorname{Det} \bar{q}_{b}^{a}} \int D E^{a} \delta\left[\sigma_{a}^{\alpha} E^{a}+\chi^{\alpha}\right]\left(\operatorname{Det} \bar{q}_{b}^{a}\right),
\end{aligned}
$$

where now the ghost operator $\mathcal{F}_{\beta}^{\alpha}$ is a gauge-fixed version of the degenerate $Q_{\beta}^{\alpha}$,

$$
\mathcal{F}_{\beta}^{\alpha}=Q_{\beta}^{\alpha}+\sigma_{a}^{\alpha} \omega_{\beta}^{a} .
$$

The determinants of matrices

$$
\begin{aligned}
& q_{b}^{a}=\omega_{\alpha}^{a} Z_{b}^{\alpha}, \\
& \bar{q}_{b}^{a}=\bar{Z}_{\alpha}^{a} \sigma_{b}^{\alpha}
\end{aligned}
$$

represent the Faddeev-Popov ghost factors for the original ghost fields (note that their double nature corresponds to two different zero modes of $Q_{\beta}^{\alpha}$ - its right and left zero vectors). A simple exercise using these zero modes shows that the ratio of determinants in (5.9) is independent of the choice of gauge parameters $\sigma_{b}^{\alpha}$ and $\omega_{\alpha}^{a}$ (see Appendix A)

$$
\delta_{(\omega, \sigma)} \frac{\operatorname{Det} \mathcal{F}_{\beta}^{\alpha}}{\operatorname{Det} q_{b}^{a} \operatorname{Det} \bar{q}_{b}^{a}}=0 .
$$

Also in the case of a redundant set of gauge conditions (5.3)

$$
\delta_{\sigma}\left\{\delta\left[\sigma_{a}^{\alpha} E^{a}+\chi^{\alpha}\right]\left(\operatorname{Det} \bar{q}_{b}^{a}\right)\right\}=0 .
$$

This makes the ghost sector of the path integral independent of the choice of functions $\left(\sigma_{b}^{\alpha}, \omega_{\alpha}^{a}\right)$ fixing additional gauge symmetries associated with the linear dependence of generators. 


\section{Gauge and ghost sector}

We now apply the above procedure to our model. First note that the range of the index $a$ here is just one discrete value corresponding to one linear dependence relation (4.15), so that we will omit this index at al. Regarding additional gauge parameters $\sigma_{b}^{\alpha}=\sigma^{\alpha}$ and $\omega_{\alpha}^{a}=\omega_{\alpha}$, there exists a convenient choice which renders the Faddeev-Popov operator (5.10) a block-diagonal structure. They read as the following 2-dimensional column and row

$$
\sigma^{\alpha}=\left[\begin{array}{c}
\sigma(\tau) \\
1
\end{array}\right], \quad \omega_{\alpha}=\left[\begin{array}{cc}
\frac{g^{2}}{a} & 0
\end{array}\right]
$$

and in view of (4.19) yield the operator

$$
\mathcal{F}_{\beta}^{\alpha}=\left[\begin{array}{cc}
\mathcal{F}\left(\tau_{1}, \tau_{2}\right) \frac{1}{a_{2}} & 0 \\
0 & Q
\end{array}\right], \quad \mathcal{F}\left(\tau, \tau^{\prime}\right)=-\frac{d^{2}}{d \tau^{2}} \delta\left(\tau, \tau^{\prime}\right)+\sigma(\tau) g^{2}\left(\tau^{\prime}\right) .
$$

The function $\sigma(\tau)$ is rather arbitrary, and should be chosen to guarantee the invertibility of the corresponding ghosts for ghosts operators

$$
\begin{aligned}
& \bar{q} \equiv \bar{Z}_{\alpha} \sigma^{\alpha}=\oint d \tau \sigma(\tau), \\
& q \equiv \omega_{\alpha} Z^{\alpha}=\oint d \tau g^{2}(\tau)=Q .
\end{aligned}
$$

This is easily attained with a generic positive-definite $\sigma(\tau)$. Therefore, the calculation of the determinant of (6.2) reduces to that of Det $\mathcal{F}\left(\tau, \tau^{\prime}\right)$ which can be recovered from the variational equation

$$
\delta_{\sigma} \ln \left[\operatorname{Det} \mathcal{F}\left(\tau, \tau^{\prime}\right)\right]=\oint d \tau_{1} \delta \sigma_{1} \oint d \tau_{2} g_{2}^{2} G\left(\tau_{2}, \tau_{1}\right) .
$$

Here $G\left(\tau_{2}, \tau_{1}\right)=\delta f\left(\tau_{2}\right) / \delta J\left(\tau_{1}\right)$ is the inverse of the operator $\mathcal{F}\left(\tau_{1}, \tau_{2}\right)$ or the Green's function of the problem

$$
-f^{\prime \prime}(\tau)+\sigma(\tau) \oint d \tau_{1} g^{2}\left(\tau_{1}\right) f\left(\tau_{1}\right)=J(\tau)
$$

for the function $f(\tau)$ periodic on a circle (with periodic derivatives). In view of the periodicity of $f^{\prime}$ the integration of this equation gives $\oint d \tau g^{2} f=\oint d \tau J(\tau) / \bar{q}$, so that the variation of the functional determinant above reads

$$
\begin{aligned}
\delta_{\sigma} \ln \left[\operatorname{Det} \mathcal{F}\left(\tau, \tau^{\prime}\right)\right] & =\left.\oint d \tau g^{2} f\right|_{J=\delta \sigma} \\
& =\frac{1}{\bar{q}} \oint d \tau \delta \sigma(\tau)=\delta_{\sigma} \ln \bar{q} .
\end{aligned}
$$

The functional dependence of this determinant on $g(\tau)$ follows from a similar variation

$$
\begin{aligned}
\delta_{g} \ln \left[\operatorname{Det} \mathcal{F}\left(\tau, \tau^{\prime}\right)\right] & =\oint d \tau_{1} \sigma_{1} \oint d \tau_{2} \delta\left(g_{2}^{2}\right) G\left(\tau_{2}, \tau_{1}\right) \\
& =\left.\oint d \tau \delta\left(g^{2}\right) f\right|_{J=\sigma}=\delta_{g} \ln Q .
\end{aligned}
$$

Here we have used the fact that the solution of the problem (6.6) for a special choice of the source $J(\tau)=\sigma(\tau)$ reads as $\left.f\right|_{J=\sigma}=1 / Q$. Indeed, with this choice $\oint d \tau g^{2} f=1$, and the resulting equation $f^{\prime \prime}=0$ has as a periodic solution the constant - the inverse of $\oint d \tau g^{2}=Q$. Therefore

$$
\operatorname{Det} \mathcal{F}\left(\tau, \tau^{\prime}\right)=\text { const } \times \bar{q} Q,
$$


so that the ratio of determinants $\operatorname{Det} \mathcal{F}_{\beta}^{\alpha} / \operatorname{Det} q_{b}^{a} \operatorname{Det} \bar{q}_{b}^{a}$ in (5.9) which can be regarded as the gaugeindependent definition of the restricted functional determinant $\operatorname{Det}_{*} \boldsymbol{Q}$ of (2.2) reads as

$$
\operatorname{Det}_{*} \boldsymbol{Q} \equiv \frac{\operatorname{Det} \mathcal{F}_{\beta}^{\alpha}}{q \bar{q}}=\text { const } \times Q\left(\prod_{\tau} a(\tau)\right)^{-1} .
$$

The delta function of gauge conditions (4.18) modified by the extra ghost $E^{a}=E$ with the choice of gauge parameters (6.1) reads as

$$
\delta\left[\sigma^{\alpha} E+\chi^{\alpha}\right]=\delta(E+\oint d \tau g \varphi) \delta\left[n^{\prime}(\tau)+\sigma(\tau) E\right] .
$$

The support of the second delta function here is given by the solution of the equation $n^{\prime}(\tau)+\sigma(\tau) E=0$, which implies in view of the periodicity of $n(\tau)$ and non-degeneracy of $\bar{q}$, that separately $E=0$ and $n^{\prime}=0$. Therefore the functional integral over $n(\tau)$ of this delta function with any functional $\Phi[n(\tau)]$ should reduce to the ordinary integral over the constant mode of $n(\tau), n_{0}=$ const, and be proportional to $\delta(E)$. As shown in the Appendix B this is indeed the case

$$
\int \operatorname{DndE} \delta\left[n^{\prime}(\tau)+\sigma(\tau) E\right] \Phi[n(\tau), E] \bar{q}=\mathrm{const} \times T \int_{-\infty}^{+\infty} d n_{0} \Phi\left[n_{0}, 0\right] .
$$

This, in particular, confirms the relation (5.14).

\section{Metric variables sector}

We apply now the path integral (5.9) to our model with $S[\phi]=\Gamma_{(2)}[\varphi, n]$. Using the expressions for the gauge-fixed ghost contribution (6.10) and the delta function of the full set of gauge conditions (6.11) in this integral we have on account of 6.12)

$$
\begin{aligned}
P=\int & D \phi D \Phi_{\mathrm{g}} \exp \left(-S_{\mathrm{eff}}\right) \\
& =\int D \varphi D n \exp \left(-\Gamma_{(2)}[\varphi, n]\right) \frac{\operatorname{Det} \mathcal{F}_{\beta}^{\alpha}}{q} \int d E \delta\left[\sigma^{\alpha} E+\chi^{\alpha}\right] \\
& =\operatorname{const} \times T \int_{-\infty}^{\infty} d n_{0} \int D \varphi \exp \left(-\Gamma_{(2)}\left[\varphi, n_{0}\right]\right) \delta(\oint d \tau g \varphi) Q .
\end{aligned}
$$

Here the local factor in the canonical integration measure [15]

$$
D \phi=D(\delta a) D(\delta N) \prod_{\tau}|\mathcal{D}(\tau)|^{1 / 2}=D \varphi D n \prod_{\tau} a(\tau)
$$

gets canceled by the local factor of (6.10), and also all factors of $\bar{q}$ cancel out, as they should due to the gauge independent nature of the gauge-fixing procedure.

With the quadratic action (4.9) essentially simplified for a constant $n(\tau)=n_{0}$, this integral takes the form

$$
P=T \int_{-\infty}^{\infty} d n_{0} \exp \left\{\frac{1}{2} n_{0}^{2}\left(-\varepsilon_{\mathcal{D}} Q-\frac{d^{2} F}{d \eta^{2}} T^{2}\right)\right\} K\left(n_{0}\right)
$$

where $Q$ is just the Faddeev-Popov factor (2.5), $T$ is a full period (4.10) of the Euclidean time and $K\left(n_{0}\right)$ is the following path integral

$$
K\left(n_{0}\right)=\int D \varphi \delta(\oint d \tau g \varphi) Q \exp \left\{-\varepsilon_{\mathcal{D}} \oint d \tau\left(\frac{1}{2} \varphi \boldsymbol{F} \varphi+2 n_{0} g^{\prime} \varphi\right)\right\} .
$$


Here $\boldsymbol{F}$ is the operator (2.15) - the kernel of the quadratic in $\varphi$ part of $\Gamma_{(2)}[\varphi, n]$. It has as a zero mode the function $g(\tau)$ - the generator of the residual transformation $\Delta^{\varepsilon} \varphi=\varepsilon g$. In fact $K\left(n_{0}\right)$ is the Faddeev-Popov path integral with this residual transformation gauged out by the auxiliary gaug $\Xi_{-}$ the lower entry of (4.18). Note that, modulo the source term linear in $\varphi$, this path integral is just the definition of the functional determinant of the degenerate operator $\boldsymbol{F}$ on the subspace of its non-zero eigenmodes, introduced in (1.3), $\left(\operatorname{Det}_{*} \boldsymbol{F}\right)^{-1 / 2}$.

Representing the delta function of this gauge via the integral over the Lagrangian multiplier $\pi$ we get the Gaussian path integral over $\Phi=(\varphi(\tau), \pi)$ with the new effective action

$$
\begin{gathered}
K\left(n_{0}\right)=\left.Q \int D \Phi \exp \left(-\varepsilon_{\mathcal{D}} S_{\mathrm{eff}}[\Phi ; J]\right)\right|_{J=-2 n_{0} g^{\prime}} \\
=\left.Q(\operatorname{Det} \mathbb{F})^{-1 / 2} \exp \left(-\varepsilon_{\mathcal{D}} S_{\mathrm{eff}}[\Phi ; J]\right)\right|_{\text {on shell }}, \\
S_{\mathrm{eff}}[\Phi ; J]=\oint d \tau\left(\frac{1}{2} \varphi \boldsymbol{F} \varphi-i \pi g \varphi-J \varphi\right) .
\end{gathered}
$$

Here $\mathbb{F}$ is the Hessian of this action (up to a sign factor $\varepsilon_{\mathcal{D}}= \pm 1$ ) with respect to the function $\varphi(\tau)$ and the numerical variable $\pi$

$$
\mathbb{F}=\frac{\delta^{2} S_{\text {eff }}}{\delta \Phi_{1} \delta \Phi_{2}}=\left[\begin{array}{cc}
\boldsymbol{F} \delta\left(\tau_{1}, \tau_{2}\right) & -i g\left(\tau_{1}\right) \\
-i g\left(\tau_{2}\right) & 0
\end{array}\right]
$$

(note the position of time entries associated with the variables $\Phi_{1}=\left(\varphi\left(\tau_{1}\right), \pi\right)$ and $\Phi_{2}=\left(\varphi\left(\tau_{2}\right), \pi\right)$ ), and the onshell condition here implies the evaluation of the action at its stationary configuration the periodic solution of the following variational problem with the source $J=-2 n_{0} g^{\prime}$,

$$
\begin{aligned}
& \boldsymbol{F} \varphi(\tau)-i \pi g(\tau)-J(\tau)=0, \\
& i \oint d \tau g \varphi=0 .
\end{aligned}
$$

\subsection{The solution for the metric perturbation and its action}

Multiplying Eq.(7.8) by $g(\tau)$ and integrating by parts one finds on account of $\boldsymbol{F} g=0$ the value of $\pi$, $\pi=-i \oint d \tau g J / Q$, and a new equation for $\varphi$ with a modified source $\tilde{J}(\tau)$

$$
\begin{aligned}
& \boldsymbol{F} \varphi(\tau)=\tilde{J}(\tau), \\
& \tilde{J}(\tau) \equiv J(\tau)-\frac{g(\tau)}{Q} \oint d \tau_{1} g\left(\tau_{1}\right) J\left(\tau_{1}\right), \quad \oint d \tau g \tilde{J} \equiv 0 .
\end{aligned}
$$

This source $\tilde{J}(\tau)$ is functionally orthogonal to the zero mode $g(\tau)$ - the property that guarantees the existence of the solution of this equation whose left hand side is also orthogonal to $g$. Thus, the problem reduces to the solution of

$$
\begin{gathered}
\boldsymbol{F} \tilde{\varphi}(\tau)=\tilde{J}(\tau), \\
\oint d \tau g \tilde{\varphi}=0,
\end{gathered}
$$

in terms of which the on shell exponential in (7.5) reads

$$
\left.S_{\text {eff }}[\varphi(\tau), \pi ; J]\right|_{\text {on shell }}=\left.n_{0} \oint d \tau \tilde{\varphi} g^{\prime}\right|_{J=-2 n_{0} g^{\prime}}
$$

\footnotetext{
${ }^{5}$ Note that even the presence of the source term for $\varphi$ in the action does not break its invariance under the $\varepsilon$ transformation, because for a constant $n_{0}$ it transforms by a total derivative term $\sim g g^{\prime}$.
} 
Below we present the solution $\tilde{\varphi}$ along with the Green's function of the problem (7.12)-(7.13), which give the answer for the exponential and preexponential factor of $K\left(n_{0}\right)$.

For this we will need an explicit parametrization of the $\tau$-range associated with the oscillatory nature of the functions $g(\tau)$ and $a(\tau)$. It was introduced in Sect.2 in the form of a circle of the circumference $T=2\left(\tau_{+}-\tau_{-}\right), \tau_{-} \equiv 0$, parameterized by $\tau$ in the range $-\tau_{+} \leq \tau \leq \tau_{+}$with the points $\pm \tau_{+}$identified. The antipodal points on the circle $\tau_{ \pm}$are distinguished by the fact that they represent two first degree zeros of $g(\tau)$ and correspond to the maximum and minimum of the scale factor.

Then the solution of (7.12)-(7.13) can be looked for as a linear combination of the partial solution of the inhomogeneous equation (7.12)

$$
\Phi(\tau)=-g(\tau) \int_{0}^{\tau} \frac{d y}{g^{2}(y)} \int_{0}^{y} d \tau^{\prime} g \tilde{J}\left(\tau^{\prime}\right)=-\int_{0}^{\tau} d \tau^{\prime} \Psi\left(\tau, \tau^{\prime}\right) g\left(\tau^{\prime}\right) \tilde{J}\left(\tau^{\prime}\right) .
$$

and the two basis functions of $\boldsymbol{F}$ - the periodic function $g(\tau)$ and the non-periodic $\Psi\left(\tau, \tau_{*}\right)$ with some $\tau^{\prime}=\tau_{*}>0$, defined by (2.18). For a negative $\tau$ the role of this second basis function will be played by $\Psi\left(-\tau, \tau_{*}\right)$ which also satisfies the equation $\boldsymbol{F} \Psi\left(-\tau, \tau_{*}\right)=0$ in view of the odd nature of $g(\tau)$. The role of these basis functions is to fix the lack of periodicity of the partial solution $\Phi(\tau)$, which itself is continuous on a circle, $\Phi\left(-\tau_{+}\right)=\Phi\left(\tau_{+}\right)$(this is guaranteed by the orthogonality of the source to $g$, $\oint d \tau g \tilde{J}=0)$, but its derivative is discontinuous at $\tau= \pm\left(\tau_{+}-0\right), \Phi^{\prime}\left(\tau_{+}\right)-\Phi^{\prime}\left(-\tau_{+}\right) \neq 0$. As shown in the accompanying paper [20] the solution of (7.12)-(7.13) reads

$$
\tilde{\varphi}(\tau)=\Phi(\tau)+C \Psi\left(|\tau|, \tau_{*}\right)+D_{+} g(\tau) \theta(\tau)+D_{-} g(\tau) \theta(-\tau),
$$

where

$$
\begin{aligned}
& C=-\frac{1}{2} \frac{\Psi_{+}}{\Psi_{+} \Psi_{+}^{\prime}-\Psi_{-} \Psi_{-}^{\prime}}\left(\Phi^{\prime}\left(\tau_{+}\right)-\Phi^{\prime}\left(-\tau_{+}\right)\right), \\
& D_{ \pm}=-\frac{1}{Q} \oint d \tau g(\tau) \Phi(\tau) \mp \frac{1}{2} \Psi_{+} \frac{\Psi_{-} \Psi_{-}^{\prime}}{\Psi_{+} \Psi_{+}^{\prime}-\Psi_{-} \Psi_{-}^{\prime}}\left(\Phi^{\prime}\left(\tau_{+}\right)-\Phi^{\prime}\left(-\tau_{+}\right)\right)
\end{aligned}
$$

Here $\Psi_{ \pm}$and $\Psi_{ \pm}^{\prime}$ are given by (2.21), and in the denominators of the above expressions one easily recognizes the basic ingredient (2.20), $\boldsymbol{I}$, of our final result for the one-loop statistical sum.

The knowledge of $\tilde{\varphi}(\tau)$ allows one to find the exponential of (7.14). With the source $J=-2 n_{0} g^{\prime}$ we have

$$
\Phi(\tau)=n_{0} g(\tau) \tau, \quad C=\varepsilon_{\mathcal{D}} n_{0} \frac{T}{\boldsymbol{I}},
$$

whereas the $D$-terms of (7.16) do not contribute to this quantity. What remains finally readsin view of the symmetry $g^{\prime}(\tau)=g^{\prime}(-\tau)$ as

$$
\begin{aligned}
\left.S_{\text {eff }}[\varphi(\tau), \pi ; J]\right|_{\text {on shell }} & =n_{0} \oint d \tau g^{\prime} \Phi+2 n_{0} C \int_{0}^{\tau_{+}} d \tau g^{\prime} \Psi\left(\tau, \tau_{*}\right) \\
& =-\frac{1}{2} n_{0}^{2}\left(Q+\varepsilon_{\mathcal{D}} \frac{T^{2}}{\boldsymbol{I}}\right) .
\end{aligned}
$$

The degeneration of $\boldsymbol{I}$ to zero obviously leads to singularity of the coefficients $C$ and $D_{ \pm}$above and indicates the presence of an additional zero mode of the operator $\boldsymbol{F}$. As shown in [20] for $\boldsymbol{I}=0$, indeed, the function $\boldsymbol{\Psi}(\tau)=\Psi\left(|\tau|, \tau_{*}\right)-2 \Psi_{-} \Psi_{-}^{\prime} \theta(-\tau) g(\tau)$ turns out to be the second zero mode of $\boldsymbol{F}$. However, this mode does not generate any additional residual symmetry of the action because the source term for $\varphi$ in the action of (7.4) is not invariant under the shift of $\varphi(\tau)$ by $\boldsymbol{\Psi}(\tau)$, $\oint d \tau g^{\prime}(\tau) \boldsymbol{\Psi}(\tau)=-T / 2 \neq 0$. Therefore, no additional gauge fixing is needed, and the prefactor will stay well defined also in the limit of $\boldsymbol{I} \rightarrow 0$, as we will shortly see below. 


\subsection{One-loop prefactor from metric perturbations}

The derivation of the prefactor is done in much detail in the accompanying paper 20. Here we only present main steps of this derivation. For this we need the Green's function $\mathbb{G}$ of the matrix valued operator (7.7), $\mathbb{F} \mathbb{G}=\mathbb{I}$. It reads

$$
\mathbb{G}=\left[\begin{array}{cc}
G\left(\tau, \tau^{\prime}\right) & \frac{i g(\tau)}{Q} \\
\frac{i g\left(\tau^{\prime}\right)}{Q} & 0
\end{array}\right],
$$

where the Green's function in the diagonal block satisfies the system of equations

$$
\begin{aligned}
& \boldsymbol{F} G\left(\tau, \tau^{\prime}\right)=\delta\left(\tau, \tau^{\prime}\right)-\frac{g(\tau) g\left(\tau^{\prime}\right)}{Q}, \\
& \oint d \tau g(\tau) G\left(\tau, \tau^{\prime}\right)=0,
\end{aligned}
$$

indicating that it is the inverse of the operator $\boldsymbol{F}$ on the subspace orthogonal to its zero mode. One can show [20] that it directly expresses in terms of the Green's function $\tilde{G}\left(\tau, \tau^{\prime}\right)$ of the problem (7.12)-(7.13),

$$
G\left(\tau, \tau^{\prime}\right)=\tilde{G}\left(\tau, \tau^{\prime}\right)-\oint d \tau_{1} \tilde{G}\left(\tau, \tau_{1}\right) \frac{g\left(\tau_{1}\right) g\left(\tau^{\prime}\right)}{Q} .
$$

The latter, in its turn, can be read off (7.16), because $\Phi, C$ and $D_{ \pm}$are all linear in $\tilde{J}, \tilde{G}\left(\tau, \tau^{\prime}\right)=$ $\delta \tilde{\varphi}(\tau) / \delta \tilde{J}\left(\tau^{\prime}\right)[20$.

This Green's function (7.21) allows one to find a one-loop prefactor of (7.5) via the variational equation $\delta \ln \operatorname{Det} \mathbb{F}=\operatorname{Tr}(\delta \mathbb{F} \mathbb{G})$. A rather lengthy calculation of this quantity in 20 gives $\delta \ln$ Det $\mathbb{F}=$ $\delta \ln |\boldsymbol{I}|+2 \delta \ln Q$ with $\boldsymbol{I}$ defined by (2.20). Therefore the prefactor of $K\left(n_{0}\right)$, which actually serves as the definition of the restricted functional determinant of $\boldsymbol{F}$ introduced in Sect.2, equals

$$
\left(\operatorname{Det}_{*} \boldsymbol{F}\right)^{-1 / 2} \equiv(\operatorname{Det} \mathbb{F})^{-1 / 2} Q=\text { const } \times|\boldsymbol{I}|^{-1 / 2} .
$$

This implies the relation (2.8) 20]. Assembling (7.20) $)$ and (7.25) in the expression (7.5) for $K\left(n_{0}\right)$ and substituting into (7.3) we see that the $Q$-term of (7.20) cancels a similar term in the exponential of (7.3) and

$$
P=|\boldsymbol{I}|^{-1 / 2} \int_{-\infty}^{\infty} d\left(n_{0} T\right) \exp \left\{\frac{1}{2} n_{0}^{2} T^{2}\left(\frac{1}{\boldsymbol{I}}-\frac{d^{2} F}{d \eta^{2}}\right)\right\}=\text { const }\left|1-\boldsymbol{I} \frac{d^{2} F}{d \eta^{2}}\right|^{-1 / 2},
$$

which confirms the gauge-independent status of the calculational procedure and finally proves the main result (2.7)-(2.9). Also, this result remains finite for $\boldsymbol{I}=0$, which eliminates the necessity to gauge out the second zero mode of $\boldsymbol{F}$ mentioned above 6 .

\section{Conclusions}

Thus we have derived the closed algorithm for the one-loop contribution to the statistical sum of a generic time-parametrization invariant gravitational model with the Friedman-Robertson-Walker (FRW) metric. The universality of this algorithm follows from the fact that the local part of the effective action (1.5) is given by a generic Lagrangian $\mathcal{L}\left(a, a^{\prime}\right)$ only restricted by the condition that

\footnotetext{
${ }^{6}$ Note that the degeneration of $\boldsymbol{I}$ to zero implies in view of 7.25 that $\operatorname{Det}_{*} \boldsymbol{F}=0$ in full accordance with the fact that the starred determinant includes the vanishing eigenvalue of this zero mode $\boldsymbol{\Psi}(\tau)$ different from $g(\tau)$.
} 
it does not contain higher derivatives of $a(\tau)$, and the nonlocal part $F(\oint d \tau N / a)$ is a generic function of the conformal invariant - the circumference of the periodic history measured in units of the conformal time. A universal feature of the formalism is the fact that the quadratic part of the action is parameterized by one function $g(\tau)$ which is both the zero mode and the generator of the residual conformal Killing symmetry (4.11) - inalienable feature of any FRW metric. This situation, which is caused by a distinguished role of this metric - the minisuperspace sector of the theory, results in the merger of two symmetries at the overlap of two local groups - diffeomorphisms and conformal transformations. Their treatment within the gauge-fixing procedure invokes the Batalin-Vilkovisky quantization method for systems with linearly dependent generators [19. It leads to the one-loop prefactor expressed via the restricted functional determinant of the quantum-mechanical operator with the zero mode gauged out. For the latter we derive in quadratures a closed algorithm in terms of the zero mode of the above type 20 . The application of this algorithm to the concrete model the CFT driven cosmology suggested in [1, 2] - will be presented in the sequel to this paper [1].

The formalism of the above type has been developed for a limited set of saddle-point solutions having one oscillation of the cosmological scale factor. This restriction is currently explained by the fact that the major ingredient of our result - the restricted functional determinant of the operator $\boldsymbol{F}$ is known in the form (2.8) only for this simplest set of instantons - see the derivation in the accompanying paper [20]. However, the path integral applications in cosmology driven by a conformal field theory suggest cosmological instantons with arbitrary number of oscillations of the cosmological scale factor, corresponding to numerous nodes of the oscillating zero mode $g(\tau)$ [1. In particular, for the number of these oscillations tending to infinity this CFT driven cosmology approaches a new quantum gravity scale - the maximum possible value of the cosmological constant [1, 2] - where the physics and, in particular, the effects of the quantum prefactor become very interesting and important. Thus the extension of the above results to an arbitrary number of oscillations of $a(\tau)$ becomes important, as this extension - the subject of our further study - might be relevant to the cosmological constant problem.

\section{Acknowledgements}

I am indebted to A.Yu.Kamenshchik for fruitful discussions and, especially, for clarifying the situation with the second zero mode of the dynamical operator. I wish to express my gratitude to G.Dvali for hospitality at the Physics Department of the Ludwig-Maximilians University in Munich where this work was supported in part by the Humboldt Foundation. This work was also supported by the RFBR grant No 11-01-00830.

\section{Appendix A. Ward identities and gauge independence in re- ducible gauge theories}

Here we prove the gauge-independence relations (5.13)-(5.14) of a generic ghost-for-ghost gauge fixing procedure. The variation of the numerator of (5.13) follows from

$$
\delta_{\sigma} \ln \operatorname{Det} \mathcal{F}_{\beta}^{\alpha}=G_{\alpha}^{\beta} \delta \sigma_{a}^{\alpha} \omega_{\beta}^{a},
$$

where $G_{\alpha}^{\beta}$ is the inverse of the gauge-fixed ghost operator

$$
\mathcal{F}_{\nu}^{\alpha} G_{\beta}^{\nu}=\delta_{\beta}^{\alpha} \text {. }
$$

Contracting this equation with $\bar{Z}_{\alpha}^{a}$ and taking into account that this is a left zero-vector of $Q_{\nu}^{\alpha}$ we have $\bar{q}_{b}^{a} \omega_{\nu}^{b} G_{\beta}^{\nu}=\bar{Z}_{\beta}^{a}$. Therefore we have the Ward identity for the Green's function of the ghost operator involving the inverse of the ghost-for-ghost operator (5.12)

$$
\left.\omega_{\beta}^{a} G_{\alpha}^{\beta}=\left(\bar{q}^{-1}\right)_{b}^{a} \bar{Z}_{\alpha}^{b} \cdot\right)
$$


Using this in (A.1) gives

$$
\delta_{\sigma} \ln \operatorname{Det} \mathcal{F}_{\beta}^{\alpha}=\delta_{\sigma} \bar{q}_{a}^{b}\left(\bar{q}^{-1}\right)_{b}^{a}=\delta_{\sigma} \ln \operatorname{Det} \bar{q}_{b}^{a},
$$

which proves the $\sigma$-independence in (5.13). The proof of $\omega$-independence is similar and is based on the Ward identity complementary to (A.3),$G_{\alpha}^{\beta} \sigma_{a}^{\alpha}=\left(q^{-1}\right)_{a}^{b} Z_{b}^{\beta}$, which in its turn follows from contracting the alternative equation for $G_{\beta}^{\alpha}, G_{\nu}^{\alpha} \mathcal{F}_{\beta}^{\nu}=\delta_{\beta}^{\alpha}$ with the right zero-vector $Z_{b}^{\beta}$.

To prove (5.14) note that the redundant set of gauge conditions in this relation can formally be rewritten in terms of independent variables $E^{A}$ as linear combinations $\chi^{\alpha}=\chi_{A}^{\alpha} E^{A}$, where the coefficients identically satisfy the relations $\bar{Z}_{\alpha}^{a} \chi_{A}^{\alpha} \equiv 0$ with the left zero-eigenvalue eigenvectors of the (degenerate) ghost operator $Q_{\beta}^{\alpha}$. Therefore, the gauge conditions (corrected by extra ghosts) $\phi^{\alpha} \equiv \sigma_{a}^{\alpha} E^{a}+\chi^{\alpha}$ in (5.9) are in one to one correspondence with $\left(E^{a}, E^{A}\right)$ and

$$
E^{a}=\left(\bar{q}^{-1}\right)_{b}^{a} \bar{Z}_{\alpha}^{b} \phi^{\alpha} .
$$

The variables $E^{A}$ and the relevant coefficients $\chi_{A}^{\alpha}$ are independent of gauge-fixing procedure for $Q_{\beta}^{\alpha}$, i. e. of the choice of $\sigma_{a}^{\alpha}$-parameters, and

$$
\begin{aligned}
\delta_{\sigma}\left(\delta\left[\phi^{\alpha}\right]\right) & =\delta\left[E^{a}\right] \delta\left[E^{A}\right] \delta_{\sigma}\left(\frac{\partial\left(\phi^{\alpha}\right)}{\partial\left(E^{a}, E^{A}\right)}\right)^{-1} \\
& =-\delta\left[\phi^{\alpha}\right] \delta_{\sigma} \ln \operatorname{Det}\left[\sigma_{a}^{\alpha}, \chi_{A}^{\alpha}\right]=-\delta\left[\phi^{\alpha}\right] \delta \sigma_{a}^{\alpha} \frac{\partial E^{a}}{\partial \phi^{\alpha}}=\delta\left[\phi^{\alpha}\right] \delta_{\sigma} \ln \operatorname{Det} \bar{q}_{b}^{a}
\end{aligned}
$$

which proves the $\sigma$-independence relation (5.14).

\section{Appendix B. Delta function type redundant gauge conditions}

In practice, the reduction of $\chi^{\alpha}$ to a set of independent gauge conditions is not useful, especially in our case of interest (4.18), when such a reduction becomes nonlocal in time. A better way is an explicit integration over a subset of variables enforced by the delta function. To see this we write down the following integral with an arbitrary test functional $\Phi[n(\tau), E]$ over its functional and numerical arguments

$$
\begin{aligned}
& \int D n d E \delta\left[n^{\prime}(\tau)+\sigma(\tau) E\right] \Phi[n(\tau), E] \\
& \quad=\left.\Phi\left[\frac{\delta}{i \delta J(\tau)}, \frac{\partial}{i \partial j}\right] \int D n D \pi d E \exp \left(i S_{\mathrm{eff}}[n, \pi, E ; J, I, j]\right)\right|_{J=I=j=0}, \\
& S_{\mathrm{eff}}[n, \pi, E ; J, I, j]=\oint d \tau\left[\pi\left(n^{\prime}+\pi \sigma E\right)+J n+I \pi\right]+E j
\end{aligned}
$$

This is the Gaussian functional integral over $\Phi^{i}=(n(\tau), \pi(\tau), E)$ with the sources $(J(\tau), I(\tau), j)$ dual to their relevant integration variables in the "effective" action $S_{\text {eff }}[\Phi ; J, I, j]$. The Gaussian integration

$$
\int D \Phi \exp \left(i S_{\mathrm{eff}}[\Phi ; J, I, j]\right)=\left.\left(\operatorname{Det} \frac{\delta^{2} S_{\mathrm{eff}}}{\delta \Phi^{i} \delta \Phi^{k}}\right)^{-1 / 2} \exp \left(i S_{\mathrm{eff}}[\Phi ; J, I, j]\right)\right|_{\text {onshell }}
$$

restricts the exponentiated action to its stationary point - the solution of variational equations including

$$
\frac{\delta S_{\mathrm{eff}}}{\delta n(\tau)}=-\pi^{\prime}(\tau)+J(\tau)=0
$$


In view of the periodicity of $n(\tau)$ the solution of this equation exists only when $\oint d \tau J=0$, which means that the result of the functional integration here is expected to be of the delta-function type $\sim \delta(\oint d \tau J)$. A possible way to obtain this result is to regularize the exponential so that the Gaussian integral will get a well-defined stationary point for arbitrary sources. We will use the regularization by a small quadratic in $n(\tau)$ term vanishing in the limit $\varepsilon \rightarrow 0$,

$$
S_{\mathrm{eff}}^{(\varepsilon)}[n, \pi, E ; J, I, j]=\oint d \tau\left(\pi n^{\prime}+\pi \sigma E+J n+I \pi\right)+E j-\frac{\varepsilon}{2 i}(\oint d \tau n)^{2} .
$$

The corresponding variational equations take the form

$$
\begin{aligned}
& n^{\prime}+\sigma E+I=0, \\
& -\pi^{\prime}+J+i \varepsilon \oint d \tau_{1} n_{1}=0, \\
& \oint d \tau \pi \sigma+j=0 .
\end{aligned}
$$

Integrating the first of equations over the period of $\tau$ we immediately find

$$
E=-\frac{1}{\bar{q}} \oint d \tau I(\tau)
$$

where $\bar{q}$ is defined by (6.3), and

$$
\begin{aligned}
& n^{\prime}=-\tilde{I}, \quad \tilde{I}(\tau)=I(\tau)-\frac{\sigma(\tau)}{\bar{q}} \oint d \tau_{1} I_{1}, \quad \oint d \tau \tilde{I} \equiv 0, \\
& n=n(0)-\int_{0}^{\tau} d \tau_{1} \tilde{I}_{1} .
\end{aligned}
$$

The constant of integration follows from the observation that the integral of Eq. (B.7) over the period of $\tau$ gives $\oint d \tau J+i \varepsilon T \oint d \tau n=0$, and $n(\tau)$ finally equals

$$
n=-\int_{0}^{\tau} d \tau_{1} \tilde{I}_{1}+\frac{1}{T} \oint d \tau_{1} \int_{0}^{\tau_{1}} d \tau_{2} \tilde{I}_{2}+\frac{i}{\varepsilon T^{2}} \oint d \tau_{1} J_{1} .
$$

Then the integration of Eq.(B.7) gives $\pi(\tau)$ with the integration constant following from (B.8)

$$
\begin{aligned}
& \pi=\int_{0}^{\tau} d \tau_{1} \tilde{J}_{1}-\frac{1}{\bar{q}} \oint d \tau_{1} \sigma_{1} \int_{0}^{\tau_{1}} d \tau_{2} \tilde{J}_{2}-\frac{j}{\bar{q}}, \\
& \tilde{J}(\tau)=J(\tau)-\frac{1}{T} \oint d \tau_{1} J_{1}, \quad \oint d \tau \tilde{J} \equiv 0 .
\end{aligned}
$$

Thus, the regularized action has a unique well-defined stationary configuration, and the onshell value of the action with the source $I$ switched off, $I=0$, reads

$$
\left.i S_{\mathrm{eff}}^{(\varepsilon)}[n, \pi, E ; J, I, j]\right|_{I=0, \text { onshell }}=-\frac{1}{2 \varepsilon T^{2}}(\oint d \tau J)^{2} .
$$

Note that it is independent of the source $j$.

The preexponential factor of $(\underline{B .3})$ parametrically depends only on the function $\sigma(\tau)$ and the regularization parameter $\varepsilon$. This dependence can be obtained from the variational equation

$$
\delta_{\sigma} \ln \operatorname{Det} \frac{\delta^{2} S_{\mathrm{eff}}^{(\varepsilon)}}{\delta \Phi^{i} \delta \Phi^{k}}=\left(\delta_{\sigma} \frac{\delta^{2} S_{\mathrm{eff}}^{(\varepsilon)}}{\delta \Phi^{i} \delta \Phi^{k}}\right) G^{k i},
$$


where $G^{k i}=-\delta \Phi^{k} / \delta J_{i}$ is the Green's function of the problem (B.6)-(B.8) for the variables $\Phi^{k}=$ $(n(\tau), \pi(\tau), E)$ as functionals of their sources $J_{i}=(J(\tau), I(\tau), j)$. With the only nonvanishing component of the $\sigma$-variation - mixed functional and partial derivative with respect to $\pi(\tau)$ and $E$ respectively,

$$
\delta_{\sigma}\left(\frac{\delta}{\delta \pi(\tau)} \frac{\partial}{\partial E} S_{\mathrm{eff}}^{(\varepsilon)}\right)=\delta \sigma(\tau)
$$

we have

$$
\delta_{\sigma} \ln \operatorname{Det} \frac{\delta^{2} S_{\mathrm{eff}}^{(\varepsilon)}}{\delta \Phi^{i} \delta \Phi^{k}}=-2 \oint d \tau \delta_{\sigma}\left(\frac{\delta}{\delta \pi(\tau)} \frac{\partial}{\partial E} S_{\mathrm{eff}}^{(\varepsilon)}\right) \frac{\delta E}{\delta I(\tau)}=\frac{2}{\bar{q}} \oint d \tau \delta \sigma=2 \delta_{\sigma} \ln \bar{q},
$$

where we have used Eq.(B.9).

Similarly $\delta_{\varepsilon}\left(\delta^{2} S_{\text {eff }}^{(\varepsilon)} / \delta n_{1} \delta n_{2}\right)=i \delta \varepsilon$ and from (B.12) $\delta n_{2} / \delta J_{1}=i / \varepsilon T^{2}$, so that

$$
\delta_{\varepsilon} \ln \operatorname{Det} \frac{\delta^{2} S_{\mathrm{eff}}^{(\varepsilon)}}{\delta \Phi^{i} \delta \Phi^{k}}=-\oint d \tau_{1} d \tau_{2}, \delta_{\varepsilon}\left(\frac{\delta^{2} S_{\mathrm{eff}}^{(\varepsilon)}}{\delta n_{1} \delta n_{2}}\right) \frac{\delta n_{2}}{\delta J_{1}}=\delta_{\varepsilon} \ln \varepsilon .
$$

Collecting (B.15), (B.18) and (B.19) we obtain in the limit of $\varepsilon \rightarrow 0$ the anticipated delta function

$$
\begin{aligned}
& \int D n D \pi d E\left.\exp \left(i S_{\mathrm{eff}}^{(\varepsilon)}[n, \pi, E ; J, I, j]\right)\right|_{I=0} \\
&=\text { const } \times \frac{1}{\bar{q} \sqrt{\varepsilon}} \exp \left[-\frac{1}{2 \varepsilon T^{2}}(\oint d \tau J)^{2}\right]=\text { const } \frac{T}{\bar{q}} \delta(\oint d \tau J)
\end{aligned}
$$

Note that the result is independent of the source $j$ dual to the extra ghost $E$. Using this result in (B.1)

$$
\begin{aligned}
\int D n d E & \delta\left[n^{\prime}(\tau)+\sigma(\tau) E\right] \Phi[n(\tau), E] \\
= & \left.\operatorname{const} \frac{T}{\bar{q}} \Phi\left[\frac{\delta}{i \delta J(\tau)}, \frac{\partial}{i \partial j}\right] \int_{-\infty}^{\infty} d n_{0} \exp \left(i n_{0} \oint d \tau J\right)\right|_{J=j=0} \\
= & \operatorname{const} \frac{T}{\bar{q}} \int_{-\infty}^{\infty} d n_{0} \Phi\left[n_{0}, 0\right]
\end{aligned}
$$

we finally prove (6.12).

\section{References}

[1] A. O. Barvinsky and A. Yu. Kamenshchik, JCAP 09 (2006) 014, arXiv:hep-th/0605132 Phys. Rev. D 74 (2006) 121502, arXiv:hep-th/0611206.

[2] A. O. Barvinsky, Phys. Rev. Lett. 99 (2007) 071301, arXiv:hep-th/0704.0083.

[3] J. B. Hartle and S. W. Hawking, Phys. Rev. D 28 (1983) 2960; S. W. Hawking, Nucl. Phys. B 239 (1984) 257.

[4] A. D. Linde, JETP 60 (1984) 211; Lett. Nuovo Cim. 39 (1984) 401; V. A. Rubakov, JETP Lett. 39 (1984) 107; Ya. B. Zeldovich and A. A. Starobinsky, Sov. Astron. Lett. 10 (1984) 135.

[5] A. O. Barvinsky, C. Deffayet and A. Yu. Kamenshchik, JCAP 05 (2008) 020, arXiv:0801.2063.

[6] M. V. Fischetti, J. B. Hartle and B. L. Hu, Phys. Rev. D 20 (1979) 1757. 
[7] A. A. Starobinsky, Phys. Lett. B 91 (1980) 99.

[8] E. S. Fradkin and A. A. Tseytlin, Phys. Lett. B 134, 187 (1984); A. O. Barvinsky, A. G. Mirzabekian and V. V. Zhytnikov, gr-qc/9510037; P. O. Mazur and E. Mottola, Phys. Rev. D 64 (2001) 104022.

[9] L. S. Brown and J. P. Cassidy, Phys. Rev. D 16 (1977) 1712; I. Antoniadis, P. O. Mazur and E. Mottola, Phys. Rev. D 55 (1997) 4770, arXiv:9509169 [hep-th].

[10] A. O. Barvinsky, C.Deffayet and A.Yu.Kamenshchik, JCAP 05 (2010) 034, arXiv:0912.4604,

[11] A. O. Barvinsky, Statistical sum in the CFT driven cosmology, arXiv:1012.1896 [hep-th].

[12] A. O. Barvinsky, Phys. Lett 175 B (1986) 401.

[13] J. J. Halliwell, Phys. Rev. D 38 (1988) 2468.

[14] J. J. Halliwell and J. B. Hartle, Phys. Rev. D 43 (1991) 1170.

[15] A. O. Barvinsky, Phys. Reports 230 (1993) 237; Phys. Lett. B 428 (1998) 322, hep-th/9712001; Nucl. Phys. B 520 (1998) 533, hep-th/9711164.

[16] S. Anderegg and V. Mukhanov, Phys. Lett. B 331 (1994) 30, hep-th/9403091

[17] L. J. Garay, J. J. Halliwell and G. A. Mena Marugan, Phys. Rev. D43 (1991) 2572.

[18] A. O. Barvinsky, Phys. Lett 195 B (1987) 344.

[19] I. A. Batalin and G. A. Vilkovisky, Phys. Rev. D28 (1983) 2567.

[20] A. O. Barvinsky and A.Yu.Kamenshchik, On the functional determinant of a special operator with a zero mode in cosmology, arXiv:1012.1571 [hep-th].

[21] J. Bardeen, Phys. Rev. D 22 (1980) 1882.

[22] V. F. Mukhanov, Physical Foundations of Cosmology, Cambridge University Press, New York, 2005.

[23] A. O. Barvinsky, A. Yu. Kamenshchik, C. Kiefer, C. Steinwachs, Phys. Rev. D81 (2010) 043530, arXiv:0911.1408,

[24] G. W. Gibbons, S. W. Hawking and M. J. Perry, Nucl. Phys. B 138 (1978) 141; J. B. Hartle and K. Schleich, The conformal rotation in linearized gravity, in Quantum Field Theory and Quantum Statistics: Essays in Honour of the Sixtieth Birthday of E. S. Fradkin, eds. I. A. Batalin, C. J. Isham and G. A. Vilkovisky (Hilger, Bristol, 1987); K. Schleich, Phys. Rev. D 36 (1987) 2342; P. O. Mazur and E. Mottola, Nucl. Phys. B341 (1990) 187. 